$100 \%$ of senders and recipients strongly agreed that the initiative was a good idea and should be continued.

Conclusion Current feedback is extremely positive. Morale within the department has improved and staff have been very receptive to the scheme. 'Learning from Excellence' has highlighted the importance of focusing on positive actions as well as errors and mistakes within the healthcare environment.

\section{G84(P) HOW APPROPRIATE ARE OUR EEGS?}

${ }^{1} \mathrm{G}$ Lanz, ${ }^{2} \mathrm{U}$ O'Neill, ${ }^{2} \mathrm{D}$ Cassidy. ${ }^{1}$ Medical School, Queens University Belfast, Belfast, UK; ${ }^{2}$ Paediatric Department, Daisy Hill Hospital, Southern Health and Social Care Trust, Newry, UK

\subsection{6/archdischild-2018-rcpch.82}

Aims An increase in EEG waiting times prompted an audit of EEG referrals, using the NICE guideline, CG137, to define our audit criteria. We aimed to audit all paediatric EEG referrals made from our paediatric service, between January to December 2016.

We aimed to audit the following:

1. Was the referral appropriate i.e. was it being carried out for suspected epilepsy, or inappropriate i.e. probable syncope or non-epileptic event.

2. Was the length of time between referral and EEG being performed within 4 weeks?

3. In the case of suspected epilepsy with a normal first EEG, was a further sleep deprived EEG (SDE) requested.

4. Patient diagnosis following investigation

Methods This was a retrospective audit, using information gathered from the Northern Ireland Electronic Care Record. The local EEG department supplied a list of all EEG referrals made in 2016 from our paediatric department. For each referral, we recorded the following: indication for referral, referral waiting time, EEG type and patient diagnosis. Using this information, we were able to categorise referrals as appropriate or inappropriate.

Results 131 patients were identified by the EEG department. We excluded 36 patients with known epilepsy and 4 patients who were under 3 months at the time of their seizure, leaving 91 patient records available for the audit. Of these, 5 patients were not counted; 1 patient's information was unavailable and 4 patients did not attend for EEG or follow-up appointments. A total of 86 patient records were included in the final audit. $60.5 \%$ of EEG referrals were assessed as appropriate. $39.5 \%$ were deemed inappropriate.

$29.8 \%$ of patients had an EEG carried out within 4 weeks. The average outpatient EEG waiting time was 66 days.

17 patients with suspected epilepsy with normal EEG, went on to have a SDE.

15 patients received a new diagnosis of epilepsy.

Conclusion Our results show that EEGs are being requested for suspected non-epileptic events which leads to the risk of false positive results, increased waiting times and unnecessary tests being carried out. We recommend that further education is required to support clinicians in requesting tests judiciously.

\section{G85(P) ADMISSIONS TO HOSPITAL AND AWARENESS OF KAWASAKI DISEASE IN ENGLAND}

${ }^{1}$ AM George, ${ }^{2}$ R McCormack, ${ }^{1,2}$ RMR Tulloh. 'Department of Paediatric Cardiology, Bristol Royal Hospital for Children, Bristol, UK; ${ }^{2}$ Societi, UK Foundation for Kawasaki Disease, Newark, UK

10.1136/archdischild-2018-rcpch.83

Background Kawasaki disease (KD) is now the commonest acquired heart disease in children in the Western world. Recent studies, such as the British Paediatric Surveillance Unit study, have suggested that the incidence in the UK is now around 9.1/100,000 children under 5 years. In May 2016, a national NHS England patient safety alert (PSA) was announced. Patients with coronary artery aneurysms (CAA) due to $\mathrm{KD}$ were to be alerted to the PSA, recalled and receive a patient specific protocol. Children in the UK have amongst the poorest outcomes globally with 24\% experiencing CAA despite treatment. We wished to determine if admissions with KD increased during the last 10 years.

Methods We undertook a Freedom of Information survey of all NHS Trusts in England (who would have received the PSA), to determine admissions with KD from 2005-2016. We asked whether the PSA had been acted upon and whether patients were recalled with CAA and aware of the PSA and which guidelines were being followed.

Results A poor response was seen to the PSA with only 11 Trusts intending to inform patients of the PSA, at routine clinic visits with no active recall. The PSA was circulated to clinicians through routine channels with few cascades to relevant clinicians. 81 Trusts reported admissions had increased four-fold from 130 per year to 400 per year, $63 \%$ being male. A further $10 \%$ of Trusts have yet to reply. Of those responding, under $50 \%$ were using the published guideline from 2013, most had a local guideline or were using no guideline. Over the same period, presenting age reduced from median 2.8 years to median 1.6 years.

Conclusions There is a marked increase in admissions in the UK coded as KD over the 10 years from 2006-2015. Despite this, few Trusts have responded to the PSA and awareness of the disease and its complication rate amongst the medical community and general public remains low. Significantly increasing incidence and poor levels of awareness have resulted in late diagnosis beyond the recommended 5 days of illness, resulting in increased risks of CAA with expected long term effects.

\section{G86(P) BIOMARKERS FOR CORONARY ARTERY ANEURYSMS IN KAWASAKI DISEASE}

${ }^{1} \mathrm{AM}$ George, ${ }^{1,2} \mathrm{RMR}$ Tulloh. 'University of Bristol, Bristol, UK; ${ }^{2}$ Bristol Royal Hospital for Children, Bristol, UK

10.1136/archdischild-2018-rcpch.84

Background Kawasaki disease (KD) is now the commonest acquired heart disease in the Western world estimated in the UK at 9.1/100,000 children under 5 years. 24\% of children in the UK develop coronary artery aneurysms (CAA) despite 